\title{
Riesgo de morbilidad y mortalidad en recién nacidos de término según adecuación del peso a la edad gestacional
}

\author{
Patricio Ventura-Juncá T. '; Gabriela Juez L. '; Eduardo Lucero M. ${ }^{2}$
}

\section{Intrauterine growth, morbidity and mortality risks in term newborns}

\begin{abstract}
To ossess neonatal prognosis in terms of hospitalization rates and mortality risks in term newborn intants from their classification as small (SGA, adequate (AGA) and low [LGA] birth weight for gestational age, by established chilean intrauterine growth polterns, prospective intormation an 21725 consecurive live term newborn intants [10\% SGA; $82 \%$ AGA and $8 \%$ LGA) was analized. Neonatol mortality and hospitalization rates in SGA (1\% and 19\%) were respectively signilicantly higher $(p<0.001)$ thon among ASTA $j 0.13 \%$ and $10 \%$ and LGA $[0.23 \%$ and $13 \%$. LGA infonts had significantly highes $\{p<0.002$ ) hospitolizchion rales $[13 \%$ ) than those with AGA $110 \%$. Risk of death (2.9\%) and morbidity $(41.7 \%)$ were also significantly higher $[p<0.001)$ ir: SGA when their bith weight was below percentile 2 for gestalional age than when it was between percenliles 2 and $1010.51 \%$ and $12 \%$ respectively). AGA infants had significantly lower risks than SGA. beween 2 and 10 percentiles $\{p<0,001$ and $p<0,002$. Breakpoints in prognosis were found at 2,10 and 90 of bisth we ght for gestational age percentiles.
\end{abstract}

(Koy words: newborn, birth weight, gestational age, orogrosis.)

En un estudio anterior, que excluía los niños con causas identificables de retardo de crecimiento intrauterino (RCIU), se comparó el número de recién nacidos de término (RNT) pequeños para la edad gestacional (PEG) de 37 semanas o más ${ }^{1}$, identificados por la curva de crecimiento intrauterino (CIU) de Lubchenco $y$ por otra elaborada con niños chilenos ${ }^{2}{ }^{3}$. El núnero de RN PEG identificados con esta curva fue $11 \%$, ante $2 \%$ al usar la de Lubchenco. También se constató que los riesgos de apgar bajo, hospitalización y mortalidad entre los $\mathrm{RN}$ PEG identificados adicionalmente con la curva chilena eran significativamente mayores que en los RNT AEG'.

Posteriormente se completó la confecciôn de una curva de CIU chilena que, además, precisaba mejor la identificación de los niños PEG al posibilitar correcciones por sexo del recién na-

1. Pontificia Universidad Católica de Chile, Faculad de Medicina, Departamento de Pediatria, Unidad de Neonatología,

2. Ingeniero. Pontificia Universidad Católica de Chile, Facultad de Medicina, Departamento de Pediatría, Unidad de Neonatología.

Financiamiento: Proyecto Fondecyt 92/759. cido $(\mathrm{RN})$ y paridad y talla maternas ${ }^{4,5}$ y coincidía con otras nacionales ${ }^{6-9}$ al demostrar que, aplicando los criterios de Lubchenco en este país, se subregistraban RN PEG. En 1992 el Ministerio de Salud recomendó el uso de dicha curva para Chile.

La clasificación de los recién nacidos de acuerdo a la adecuación de su peso a la edad gestacional, según se sitúen bajo o sobre los percentiles 10 y 90 respectivamente, en pequeños (PEG) adecuados (AEG) y grandes (GEG) para la edad gestacional, ha sido considerada un jndicador útil para evaluar el riesgo de morbilidad y mortalidad ${ }^{10-13}$ y es utilizada en todas las unidades de neonatología.

Los riesgos de morbilidad y mortalidad de los niffos ubicados bajo el percentil 10 no parecen homógeneos ${ }^{14 \cdot 16}$. La aplicación de la nueva curva aumenta significativamente el número de RN PEG y no parecería apropiado tomar con todos las mismas precauciones. Es importante, por lo tanto, precisar cuáles requieren más observación y búsqueda de posibles enfermedades.

El proposito de este trabajo fue precisar y comparar los riesgos de mortalidad y de morbi- 
lidad que requiere hospitalizacion en RNT PEG, AEG y GEG, identificados con la nueva curva de crecimiento intrauterino recomendada por el Ministerio de Salud en Chile y determinar dichos riesgos según tramos de percentiles, en especial para RNT PEG.

\section{Material y Métodos}

Se utilizó una base de datos computadorizada prospectiva desarrollada en nuestra unidad de recién nacidos desde 1978. Los métodos para las mediciones antropométricas, evaluación de la edad gestacional, registro de datos clíni$\cos$ y el sistema computacional para ingreso y validación de datos, han sido descritos en varias publicaciones anteriores ${ }^{l,}$ 3-5, 17 .

Se dererminaron los percentiles $2,4,5,6$ y 8 con el archivo seleccionado, utilizado para la confección de la curva de ClU, que elimina a todos los recién nacidos con malformaciones $y$ causas conocidas de retardo de crecimiento intrauterino (tabla).

Se estudiaron 21 725 RNT PEG (37 semanas o mís) nacidos consecutivamente $y$ registrados en la base de da. tos. en el transcurso de 10 años, en la Maternidad del Hospital Clínico de la Universidad Católica. Se identificó a los RN PEG, AEG y GEG utilizando los factores de corrección por sexo del RN y talla y paridad raterna. Se identific6 el nưmero de niños que fallecieron y los que sufrieron morbilidad que requirió hospitalización. Se comparó el porcentaje de RN fallecidos $y$ hospitalizados entre estos tres grupos y luego se hizo otro tanto por tramos de percentiles.

Los nacidos de término AEG se agruparon en tramos de percentiles 5 a $10 ; 25$ a 50 ; 50 a 75 y 75 a 90 . Los nacidos de término PEG fueron ordenados en tramos de dos en dos percentiles ( $<2 ; 4$ a $6 ; 6$ a 8 y 8 a 10 ) o más amplios $(<2 ; 2$ a $6 ; 6$ a $10 ; 2$ a 5 y 5 a 10$)$. Se comparo la mortalidad y hospitalización en estos tramos de percentiles. A las comparaciones de los porcentajes de mortalidad $y$ hospitalización entre los grupos se aplicó la prueba de $\chi^{2}$. con correccion del factor de contiouidad.

\section{Resultados}

En la tabla se expresan los percentiles $2,4,5$, 6 y 8 para las edades gestacionales de 37 a 42 semanas.

Según peso y edad gestacional, 2238 (10\%) de los RN era PEG; 17775 (82\%) AEG y $1712(8 \%)$ GEG. La mortalidad de los RN PEG fue significativamente mayor $(1,03 \%$; $\mathrm{p}<$ $0,001)$ que en los AEG $(0,13 \%)$ y GEG $(0,23 \%)$, sin diferencias significativas entre los dos últimos.

La proporción de niños que requirieron hospitalización fue significativamente mayor en
Ios RN PEG $(19 \% ; \mathrm{p}<0,001)$ que en los AEG $(10 \%)$ y GEG (13\%) y en estos últimos también significativamente mayor que en los AEG ( $p<$ 0,002 ).

El porcentaje de RN AEG que requirí hospitalización, divididos en los tramos de percentiles 10 a $25: 25$ a $50 ; 50$ a 75 y 75 a 90 fluctuó entre 9,7 y $10,9 \%$ (p: ns). La mortalidad en los RN AEG fue menor en el tramo de percentil 50 a $75(0,04 \%)$ y mayor en los tramos 10 a 25 y 25 a 50 . La de este último fue de $0,19 \%$, que comparada con la del tramo de 50 a 75 percentiles es significativamente mayor $(\mathrm{p}<0,05)$.

En los grupos identificados por tramos de percentiles bajo el percentil 10 , las proporciones de fallecidos y hospitalizados fueron significativamente mayores en los ubicados bajo el percentil $2(14 / 482 ; 2,9 \%$ y $201 / 482 ; 41,7 \%$ respectivamente, $p<0,001)$ ante los del tramo de percentiles 2 a $10(23 / 17775 ; 0,51 \%$ y $1848 /$ 17775 ; $12,8 \%$ respectivamente, $\mathrm{p}<0,001$ ). En este último grupo la mortalidad y hospitalizacion fueron significativamente mayores que en el grupo de RN AEG ( $p<0,001$ y $p<0,002$ ).

La comparacion de mortalidad y hospitalización entre los grupos que se encontraban entre los percentiles 2 a 4,4 a 6,6 a 8 y 8 a 10 no mostró diferencias significativas, aunque sí una tendencia creciente a mayor porcentaje de niños hospitalizados mientras más bajo los percentiles. La mortalidad y hospitalizacion en los niños de los percentiles 2 y 6 fue de $0,5 \%$ y $14,5 \%$, entre los percentiles 6 y 10 result 6 de $0,5 \%$ y $11,5 \%$, sin diferencia significativa entre ambos grupos. Lo mismo se observó al comparar los grupos entre los percentiles 2 a 5 y 5 a 10.

\section{Tabla}

Percentiles de peso 2, 4, 5, 6 y 8 para edades gestacionales de 37 a 42 semanas

\begin{tabular}{ccccccc}
\hline $\begin{array}{l}\text { Semanas de gesta. } \\
\text { ción (semanes) }\end{array}$ & \multicolumn{5}{c}{ Pe c e n t i l e s (g) } \\
& $\mathbf{2}$ & $\mathbf{4}$ & $\mathbf{5}$ & $\mathbf{6}$ & $\mathbf{8}$ \\
\hline 37 & 2321 & 2423 & 2470 & 2496 & 2545 \\
38 & 2478 & 2590 & 2642 & 2666 & 2718 \\
39 & 2616 & 2733 & 2786 & 2809 & 2865 \\
40 & 2728 & 2848 & 2897 & 2920 & 2976 \\
41 & 2809 & 2922 & 2966 & 2991 & 3046 \\
42 & 2853 & 2954 & 2987 & 3015 & 3067 \\
\hline
\end{tabular}




\section{Comentario}

La distribución en el porcentaje de RNT PEG, AEG y GEG es algo diferente en esta serie a la de un estudio anterior con una población semejante pero sin correcciones por sexo del RN y talla y paridad materna (11\% PEG, $79,5 \%$ AEG y 9,5\% GEG)' disminuyendo proporción de PEG y GEG aumentando la de $A E G$, lo que demuestra la utilidad de la corrección mencionada para el diagnóstico más preciso de estos niños.

Las diferencias en mortalidad y hospitalización encontradas entre PEG, AEG y GEG coinciden con la de otros estudios ${ }^{10,11}$. Las cifras demuestran que los RN PEG son los de mayor riesgo.

El grupo de los RN AEG es relativamente homogéneo en riesgo de mortalidad y morbilidad que requiera hospitalizacion. En la mortalidad sólo se encontró diferencia estadísticamente significativa entre los ubicados en los percentiles 25 a 50 y 50 a 75 , que no tiene relevancia clínica debido al número pequeño de niños fallecidos en ambos subgrupos, con lo que "p" puede variar significativamente al aumentar o disminuir dicho número en uno o dos niños.

El mayor riesgo de hospitalización y morbilidad en niños bajo el percentil 2 coincide con experiencias previas sin corrección para paridad y talla materna y sexo del recién naci$\mathrm{do}^{14}$. Este es el único percentil en que se comprueba un quiebre claro en dichos riesgos y determina que estos niños requieren vigilancia clínica especial.

Los riesgos de los niños ubicados en los percentiles 2 a 10 , mayores que en los AEG respaldan la utilidad clínica de la curva de crecimiento intrauterino recomendada para Chile. Estos niños deben considerarse en riesgo moderado. El peso es poco determinante para evaluar en ellos el riesgo de morbilidad, en contraste los antecedentes perinatales y la condición al nacer.

\section{Resumen}

Se estudiaron 21725 recién nacidos de término consecutivos con el objeto de precisar los riesgos de morbilidad y mortalidad de acuerdo con su ubicación en los percentiles de adecua- ción de su peso para la edad gestacional, según las curvas de crecimiento intrauterino recomendadas por el Ministerio de Salud de Chile. Diez por ciento eran pequeños, $82 \%$ adecuados y $8 \%$ grandes para la edad gestacional. La mortalidad y la hospitalizacion resultaron significativamente mayores en los pequeños ( $1 \%$ y $19 \%$ ), que en los de peso adecuado $(0,3 \%$ y $10 \%)$ y grandes $(0,23 \%$ y $13 \%), \mathrm{p}<0,001$. La proporción de hospitalizaciones fue mayor en los recién nacidos grandes que en los de peso adecuado, $\mathrm{p}<0,002$. Los niños pequeños para la edad gestacional ubicados bajo el percentil 2 tuvieron más hospitalizaciones y mortalidad $(2,9$ y $41,7 \%$ ) que los ubicados entre los percentiles 2 y $10(0,51$ y $12,8 \%)$, p $<0,001$ y el de estos últimos mayor que en los de peso adecuado, $\mathrm{p}<0,001$. Las categorías de quiebre para riesgos significativos de morbilidad 0 mortalidad fueron los percentiles 2,10 y 90 .

(Palabras clave: recién nacido, edad gestacional, peso de nacimiento, pronóstico.)

\section{Agradecimientos}

A la labor de pediatras, obstetras, enfermeras-matronas y auxuliares de enfermería, que bicieron posible este estudio. A las secretarias Srtas. Erika Ibarra y Pamela Zunino.

\section{Agradecimientos}

1. Ventura-Juncá $P$, Juez $G$, Lucero $E$ : Desnutrición intrauterina: Identificación de una nueva población de alto riesgo con una curva de peso chilena. Rev Med Chil 1986; 114: 790.797.

2. Lubchenco L, Hansman CH, Dressier M. Boyd E: Inirauterine growth as estimated from liveborn birthweight data at 24 to 42 weeks of gestation. Pediatrics 1963; 32: 793-800.

3. Juez $G$, Ventura-Juncá $P$. Lucero $E$ : Crecimiento intrauterino en un grupo seleccionado de recién nacidos chilenos. Rev Med Chil 1984; J12: 759-764.

4. Juez $G$, Lucero $E$, Ventura-Junca $P$ : Crecimiento intrauterino en recién nacidos chilenos de clase media. Rev Chil Pedjatr 1989: 60: 198-202.

5. Juez $G$. Lucero $E$, Ventura-Juncá $P$ : Crecimiento intrauterino según sexo fetal y paridad materaa. Rev Chil Pediatr 1989; 60: 204-207.

6. Rizzardini $M$, Silva $E$, Schildlow $D$ : Curva de ctecimiento intrauterino en recién nacidos chileros. Rev Chil Pedatr 1972; 43: 7-13.

7. Krasse $S, G r o b J$, Campos G: Características antropométricas de recién nacidos vivos en Valdivia en comparación a patrón de Battaglia y Lubchenco. Rev Latin Perinat 1984; 1: 111-118. 
8. Weldt E. Rosselot B. Tohb D, Andrade C: Evaluación de crecimiento intratuterino mediante el peso de nacimiento. Rev Chil Pediatr 1988: 59: 267 269.

9. Beca JP, Rizzardini $M$. Weldt E, Fernández MP. Bloomfield $G$ : Talla, peso y perímetro cranezno según edad gestacional en recién nacidos de menos de 35 seinanas. Rev Chil Pediatr 1989; 60: 1-5.

10. Berhman $R$. Babson G. Lesel $R$ : Fetal and Neonatal Mortality risks by gestational age and weigbt. Am J Dis Child I971; 121: 486.

1 1. Lubchence L, Searis D, Bruzie J: Neonatal Monality rate: relationship to bitth weight and gestational age. I Pediat 1972; 81: 814-822.

12 Lubchenco L: Intrauterine Growth and Neonatal Morbidity and Mortality, in Lubchenco, L.O., editor, The high risk infant. WS Saunders Company, USA. 1976; 99-123.
13. Villar $J$, De Onis $M$, Kestler F, Bolaños F., Cerezo $C$. Bernedes $H$ : The differential neonatal morbjdity of the intrauterine growth retardation syndrome. Am J Obstet Gynecol 1990; 163: 151-157.

14. Balcazar $H$. Haas $J$ : Classification schemes of small for gestational age and type of intrauterine growth retardation and its implication to early neonatal mor1ality. Early Human Development 1990; 24: 219-230.

15. Ventura-Juncá P. Juez G, Escobar J: Comparación de riesgos de RNT PEG por tramos de percentiles. Rev Chil Pediatr 1992; 63 (especial): 47.

16. Kramer M. MC Lean M. Olivier M, Willis D. Usher $R$. Body proportionality and head and lenght "sparing" in growth retarded neonates: A critical reappraisal. Pediatr 1989; 84; 71?-723.

17. Juez G. Lucero E, Venfura-Juncá P: Sistema computacional de manejo de datos neonatales. Rey Chil Pediatr 1987; 58: 257-260.

\section{AVISO A LOS AUTORES}

Con el objeto de dar prioridad a los trabajos de investigación, en vista de las limitaciones de espacio de la Revista Chilena de Pediatría, el Comité Editorial ha acordado restringir la impresión de casos clínicos a un máximo de dos por cada número. 